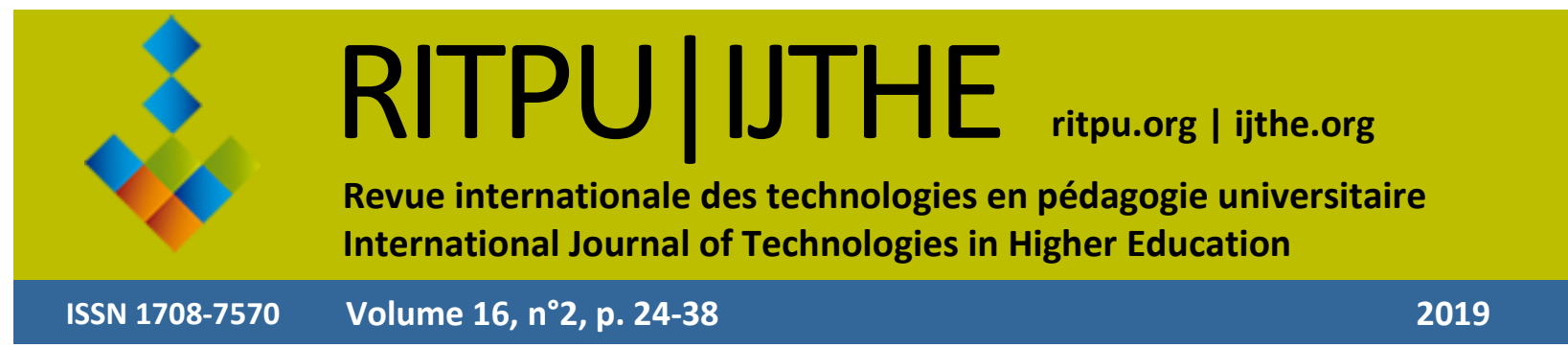

\section{Étude de compétences de référencement documentaire d'étudiants universitaires}

\section{Referencing skills of university students}

https://doi.org/10.18162/ritpu-2019-v16n2-04
Alain CADIEUX

Université du Québec en Outaouais (UQO), Canada alain.cadieux@uqo.ca

Martine PETERS

Université du Québec en Outaouais (UQO), Canada martine.peters@uqo.ca

Sarah BEAUCHEMIN-ROY Université du Québec en Outaouais (UQO), Canada sarah.beauchemin-roy@uqo.ca

\title{
Résumé
}

Les compétences de référencement documentaire font appel à des savoirs techniques, comme l'utilisation de logiciels de gestion de données bibliographiques; des savoir-faire, comme la résolution de problèmes; et des savoir-être, comme le sentiment d'autoefficacité. La présente étude explore le lien entre ces savoirs et le nombre d'années universitaires complétées. Les résultats à un questionnaire provenant de 810 étudiants québécois démontrent que ceux qui commencent leurs études universitaires sont moins efficaces et moins confiants face à leurs compétences de référencement documentaire et ont moins recours aux logiciels de gestion de données bibliographiques que ceux qui ont étudié plus longtemps. Tous les étudiants ont des savoir-faire pour résoudre leurs problèmes de référencement documentaire.

\section{Mots-clés}

Compétences, référencement documentaire, université, compétence technique, efficacité personnelle, résolution de problèmes, logiciel de gestion de données bibliographiques

\section{Abstract}

When using their referencing skills, students use three types of knowledge: 1- technical knowledge, such as the use of bibliographic data management software, 2- procedural knowledge, such as problem-solving and 3-attitudinal knowledge, such as self-efficacy. This study explores the link between referencing skills, these knowledges and the number of academic years completed by students. The results of a questionnaire from 810 Quebec students show that those who begin their university studies are less efficient and less confident in their referencing skills and have less technical knowledge with bibliographic data management software than 
those who have studied for a longer time. All students have procedural knowledge for solving problems when using their referencing skills.

\section{Keywords}

Skills, referencing skills, university, technical knowledge, self-efficacy, problem solving, bibliographic data management software

\section{Introduction}

Les compétences informationnelles, les compétences rédactionnelles et les compétences de référencement documentaire font partie des apprentissages que tous les étudiants universitaires doivent développer, notamment afin de favoriser l'intégrité académique universitaire et de prévenir le plagiat (Peters, Vincent, Gervais, Morin et Pouliot, sous presse). L'abondance de l'information et la facilité avec laquelle les étudiants y accèdent, notamment avec le Web, génèrent de nombreux défis en matière de questionnement au regard de la crédibilité, de l'utilisation et du référencement de l'information (Peters et al., sous presse). En effet, bien que les technologies permettent d'obtenir de l'information facilement pour réaliser des travaux universitaires, elles ont amené une complexité additionnelle quant au référencement de sources numériques beaucoup plus variées (ex.: blogues, forums, actes de congrès, wikis, etc.) comparativement aux formats traditionnels qui étaient utilisés auparavant dans les bibliothèques (Couture, 2010). Par exemple, il n'est pas rare de consulter une page ou un site Web où aucun auteur, année ou même titre ne soit précisé. Contrairement aux formats traditionnels de l'information (périodique, livre), la complexité à référencer ces documents s'est accentuée (Park, Mardis et Ury, 2011). Enfin, les technologies ont amené non seulement une plus grande difficulté à référencer les sources, mais aussi une plus grande fréquence de situations de plagiat (Robert, 2008), en particulier pour les étudiants qui ne savent pas comment référencer correctement leurs sources (Vardi, 2012) ou encore pour ceux qui ont des compétences rédactionnelles lacunaires, notamment en lien avec l'utilisation appropriée des sources (Vincent et Cadieux, 2019).

Les compétences de référencement peuvent faire appel à des savoirs techniques, comme l'utilisation de logiciels de gestion de données bibliographiques; des savoir-faire, comme les compétences de résolution de problèmes; et des savoir-être, comme des compétences personnelles à agir avec confiance. Dans ce contexte, il apparaît important de documenter le champ des compétences de référencement documentaire afin de cerner les différents types de savoirs qui sont mobilisés afin de permettre aux étudiants de réaliser leurs travaux universitaires avec intégrité. Bien que ces compétences se développent à tous les ordres d'enseignement, c'est à l'université qu'elles sont le plus souvent sollicitées (Newton, 2016). Ainsi, la présente étude vise à décrire comment les compétences de référencement documentaire se développent en fonction du nombre d'années d'université complétées.

\section{Problématique}

Le référencement documentaire est un «processus d'attribution explicite à un ou des auteurs de productions ou de fragments de productions, voire d'idées qui ont inspiré sa propre production » (Cadieux, Morinière et Simonnot, 2018, p. 3). Pour ce faire, l'auteur guide d'une part les lecteurs à l'intérieur d'un texte en indiquant explicitement la source des idées qu'il a empruntées à l'aide de marques spécifiques telles que les auteurs, la date et parfois la page, et doit d'autre part 
indiquer la liste des références bibliographiques à la fin du texte (Cadieux et al., 2018; Couture, 2017), cette liste permettant de retracer facilement tous les documents originaux cités dans un document. Ainsi, on peut parler de la compétence de référencement documentaire en deux temps : dans le texte, au moment de l'écriture lorsque l'auteur intègre les idées des lectures recensées ainsi qu'à la fin du texte lorsque l'auteur génère la liste de références complètes (Monney, Peters, Boies et Raymond, 2019).

Les compétences de référencement documentaire ne se limitent pas à indiquer des références dans un texte et à fournir à la fin toutes les informations requises pour retracer la source originale (Cadieux et al., 2018). En effet, comme pour les compétences rédactionnelles, les compétences de référencement démontrent que ce que l'auteur met de l'avant dans un texte a déjà été dit par d'autres auteurs dans le passé et qu'il joint leur voix à la sienne pour soutenir des arguments ou des notions de son texte (Couture, 2017). Cette démonstration peut se faire notamment en paraphrasant ou en rapportant mot à mot les paroles d'un auteur. Les compétences de référencement documentaire permettent donc de témoigner d'une recherche d'information approfondie et rigoureuse tout en restant critique face aux idées citées (Monney et al., 2019; Vardi, 2012). Comme l'exprime très bien Vardi (2012), les compétences de référencement documentaire sont complexes :

An approach that views referencing as fundamental to demonstrating critical engagement and deep understanding goes far beyond paraphrasing, quoting and summarising. Critical thinking and writing involve evaluating, analysing, interpreting and arguing - the types of higher order thinking skills that universities expect of their students. (p. 924)

Ces compétences ont été analysées dans le cadre d'une étude plus large sur l'étude des stratégies de créacollage numérique, soit les stratégies d'apprentissage faisant appel aux compétences informationnelles, rédactionnelles et de référencement documentaire pour rédiger des travaux. Le créacollage numérique est un processus utilisé par bon nombre d'étudiants qui consiste à colliger de l'information trouvée sur le Web et à la tisser dans une nouvelle création (Peters, 2015). Le contexte de cette étude a fait en sorte de cibler un certain nombre d'indicateurs dans les champs de compétences étudiés. En conséquence, la présente étude ne prétend pas faire une description exhaustive des connaissances, habiletés ou attitudes impliquées dans les compétences de référencement, mais présente plutôt les choix qui ont été faits en lien avec les écrits récents.

\section{Types de savoirs mobilisés dans les compétences de référencement}

La prochaine section présente différents types de savoirs pouvant être mobilisés au regard des compétences de référencement.

\section{Utilisation de logiciels de gestion de base de données bibliographiques}

Les logiciels de gestion de base de données bibliographiques, communément appelés « Personal bibliographic softwares, Bibliographic citation management softwares, Citation managers » (Francese, 2013, p. 145), permettent de trouver des informations, de les référencer, de les collecter dans une bibliothèque virtuelle, de les organiser et de les citer lors de la rédaction de travaux, de rapports ou d'articles scientifiques (Cuschieri, Grech et Calleja, 2019; Francese, 2013; Ram et Anbu K, 2014). Les logiciels de gestion de base de données bibliographiques facilitent la création de bibliothèques numériques qui, elles, permettent la génération automatique de listes de références, et ce, dans plusieurs formats selon le style demandé (Francese, 2013; Ram et Anbu K, 2014). Il devient ainsi possible de citer ses sources dans un 
traitement de texte afin que le logiciel de gestion de base de données bibliographiques génère automatiquement la liste de références à la fin du texte (Cuschieri et al., 2019; Francese, 2013; Ram et Anbu K, 2014).

Ces logiciels se sont grandement développés dans le monde, offrant aux usagers des versions payantes (EndNote) et d'autres complètement gratuites (Zotero, Mendeley). Bien que le développement de ces outils ait contribué à améliorer leur facilité d'utilisation, de solides connaissances technologiques de base et un soutien sont nécessaires en début d'apprentissage afin de maîtriser toutes leurs fonctions. En effet, malgré une aisance générale avec la technologie, une ambivalence semble persister chez les jeunes quant à l'utilisation des logiciels de gestion de base de données bibliographiques (Salem et Fehrmann, 2013). En fait, selon ces auteurs, il est encore possible de retrouver des étudiants qui préfèrent entrer leurs références bibliographiques manuellement (et parfois, les organiser avec un traitement de texte). Ce constat soulève la question de savoir si le manque de compétences techniques à propos des logiciels de gestion de base de données bibliographiques n'est pas lié à leur inutilisation.

Selon Speare (2018), le choix d'utiliser un logiciel de référencement est motivé par quatre principales raisons: la facilité, la gratuité, l'intégration de citations directement dans un document de traitement de texte et la présence de fonctions utiles et nécessaires. De plus, l'utilisation du logiciel est principalement motivée par les quatre fonctions suivantes : créer des listes de références pour des travaux; créer des dossiers pour organiser les citations; ajouter des citations directement dans le document de traitement de texte; sauvegarder des sources provenant des bases de données (Speare, 2018). Toutefois, Speare (2018) relève plusieurs obstacles à l'utilisation des logiciels de gestion de base de données bibliographiques, dont 1) le temps nécessaire à l'apprentissage du logiciel et ensuite, 2) le temps nécessaire à la création de la base de données, 3) la non-convivialité des interfaces, 4) le manque de confiance en soi, 5) le manque d'expérience des étudiants dans l'utilisation de logiciels de référencement et enfin, 6) le peu de références à réaliser pour un travail.

Une autre recherche menée auprès d'étudiants de $1^{\mathrm{er}}$ cycle universitaire mentionne que ceux-ci semblent trouver plus simple d'écrire leurs références manuellement plutôt que d'avoir un logiciel qui les crée pour eux (Salem et Fehrmann, 2013). Les auteurs indiquent que la plupart des participants de cette recherche semblaient indifférents ou ambivalents quant à l'utilisation d'un logiciel de gestion de base de données bibliographiques. Dans une autre recherche souhaitant mesurer le degré de confiance des participants envers plusieurs compétences liées à l'informatique, la majorité des participants se disaient confiants et expérimentés dans leur utilisation de nombreuses compétences informatiques de base et indiquaient que celles-ci étaient suffisantes pour la réalisation de travaux universitaires (Brown, Dickson, Humphreys, McQuillan et Smears, 2008). Or, toujours selon ces auteurs, les participants ont dit éprouver un manque de confiance envers leurs compétences uniquement quant à l'utilisation de logiciels de gestion de base de données bibliographiques. Ces résultats vont dans le même sens que ceux de Dahlstrom, Grunwald, de Boor et Vockley (2011) dans une recherche internationale effectuée auprès de 3000 étudiants de $1^{\text {er }}$ cycle universitaire où $51 \%$ d'entre eux avaient utilisé des outils de référencement documentaire ou des outils de citation en ligne, bien que $23 \%$ aient indiqué un manque de confiance dans leurs compétences d'utilisation de ces outils. Malheureusement, le type de programme et la discipline dans lesquels les étudiants étaient inscrits n'étaient pas précisés. Les aspects affectifs ou liés à la confiance en soi relèvent d'un deuxième type de savoir que nous aborderons ci-dessous. 


\section{Perception de sa propre efficacité}

La perception de sa propre efficacité fait référence « au niveau de confiance d'un individu dans sa capacité à mener à bien une tâche » (Bandura, 1977, p. 203). La perception de son efficacité personnelle par un individu affecte le choix de ses activités, la quantité d'efforts déployés et sa persistance dans l'une ou l'autre des nombreuses tâches de l'activité humaine, et est spécifique à un domaine de tâches (Artino, 2012). L'auteur précise que cette perception peut toutefois ne pas correspondre avec celle des autres personnes de son entourage, ce qui fait en sorte que les individus peuvent surestimer leurs habiletés. Or, plus l'individu a une perception positive et réaliste de son efficacité personnelle, plus il aura des comportements d'approche et de réussite au regard de la tâche (Tsai, 2018).

En ce qui concerne le concept de la perception de son efficacité propre en lien avec les compétences de référencement, l'étudiant apprend à attribuer certaines idées d'un texte à des sources originales. Lorsqu'il s'approprie des outils comme un guide de rédaction des références et qu'il reçoit de bons commentaires et résultats, il améliore sa confiance en lui et développe une perception positive de sa propre efficacité à référencer. En se basant sur la théorie sociocognitive d'Albert Bandura, Guerrin (2012) proposent quatre sources affectant la perception de sa propre efficacité :

1. L'expérience vécue de maîtrise, c'est-à-dire qu'un succès pourra entraîner une augmentation du sentiment d'efficacité personnelle et vice versa; tout ceci étant modulé par d'autres facteurs, dont la quantité d'efforts à déployer par l'individu (Guerrin, 2012).

2. L'observation des autres, communément nommée l'expérience vicariante, qui donne une motivation d'agir à l'individu s'il a observé quelqu'un de semblable réussir dans la tâche demandée (Guerrin, 2012).

3. La persuasion verbale d'une autre personne crédible aux yeux de l'individu sur ses propres compétences à accomplir la tâche, qui influencerait le sentiment d'autoefficacité (Guerrin, 2012).

4. L'état physiologique de l'individu, donc ses capacités physiques à accomplir la tâche demandée, qui influencerait le sentiment d'autoefficacité (Guerrin, 2012).

De ces quatre sources de motivation, l'expérience vécue de maîtrise serait la source la plus influente puisque c'est une preuve directe pour l'individu qu'il est capable de rassembler ses compétences pour y arriver (Artino, 2012).

Une fois acquise, la perception de sa propre efficacité influence le comportement et la réussite d'un individu. En effet, une méta-analyse de 100 études empiriques réalisées dans les 20 dernières années indique que des neuf constructions psychosociales couramment recherchées, le concept de perception de sa propre efficacité universitaire était le seul prédicteur significatif du rendement scolaire et de la performance des étudiants (Robbins et al., 2004, cités par Artino, 2012). Sur le plan des compétences de référencement et de l'évitement du plagiat, Newton (2016) estime qu'en fournissant aux étudiants des connaissances et des expériences positives liées au référencement et à l'évitement du plagiat, leur confiance en soi serait améliorée dans ces domaines et augmenterait ainsi leur performance. Dans son étude, Newton (2016) indique que les étudiants de cycles supérieurs (donc, avec plus de scolarité et d'expérience liées au référencement et au plagiat) étaient plus confiants dans leurs habiletés de référencement et moins indulgents envers le plagiat, et performaient mieux dans un simple test de référencement. Dans un contexte où la technologie évolue rapidement, ces qualités sont essentielles pour apprendre à 
mettre à jour ses propres connaissances et à s'adapter aux nouvelles réalités tout au long de la vie (Guerrin, 2012).

\section{Résolution de problèmes}

La résolution de problèmes peut se définir comme un processus cognitif du cerveau qui cherche une solution à un problème donné ou qui trouve un chemin pour atteindre l'objectif donné (Wang et Chiew, 2010). Selon Staats (1996), la résolution de problèmes implique essentiellement trois étapes, à savoir la détermination du problème, le raisonnement en lien avec les solutions à envisager et le comportement à adopter afin de le résoudre. En matière de référencement documentaire, les problèmes émergent, notamment, de difficultés à déterminer l'appartenance ou l'année d'une source trouvée sur Internet. Par exemple, certains textes proviennent d'actes de congrès, mais ils ont été repérés sur Internet ou dans des bases de données comme ERIC et les informations sont parfois absentes ou encore difficiles à comprendre pour les étudiants. Ainsi, il faut bien saisir toutes les particularités des sources afin de les référencer correctement.

Dans ces situations, les étudiants se tournent vers des ressources d'aide variées, ils consultent leurs amis, des enseignants ou des bibliothécaires afin de trouver des réponses à leurs interrogations et de référencer correctement les sources dans leur texte. À cet effet, une recherche qui s'intéressait à la façon dont les étudiants apprennent à citer leurs références suggère que les bibliothécaires sont une source importante d'aide, soit en personne, soit au téléphone ou en ligne (Park et al., 2011). Également, Dahlstrom et Bichsel (2014) démontrent que lorsqu'ils ont besoin de soutien technique pour des activités universitaires, la majorité des étudiants se tournent vers les ressources en ligne, dont Google et YouTube. Ils ont ensuite recours à leurs pairs, leurs amis et leur famille, particulièrement pour les plus jeunes, les plus vieux préférant utiliser le service d'assistance technique de leur université (Dahlstrom et Bichsel, 2014).

D'autres stratégies consistent à utiliser les logiciels de gestion de base de données bibliographiques. Toutefois, ceux-ci apportent leur lot de défis pour les utilisateurs, dont l'identification exacte du type de référence, le manque de standardisation des bases de données et la résistance des professeurs à l'utilisation de nouveaux styles (Park et al., 2011). À ces défis s'ajoute le besoin de connaissances informatiques pour, par exemple, la manipulation des menus des différents types et versions des logiciels, la saisie, l'utilisation et l'extraction des données de même que l'usage des différentes plateformes informatiques.

Ainsi, notre recension démontre que les compétences de référencement documentaire font appel à différents savoirs, savoir-faire et savoir-être tels que l'utilisation de logiciels de gestion de base de données bibliographiques, l'efficacité personnelle et la résolution de problèmes. Par ailleurs, les recherches ne précisent pas comment ces compétences évoluent en fonction de l'expérience universitaire de même qu'en fonction des types de programmes suivis à l'université.

\section{Objectif}

En conséquence, cette étude vise à vérifier comment l'utilisation de logiciels de gestion de base de données bibliographiques, l'efficacité personnelle et la résolution de problèmes en matière de référencement documentaire se développent en fonction du nombre d'années complétées à l'université et des types de programmes suivis par les étudiants. 


\section{Méthodologie}

Cette étude s'inscrit dans un plus grand projet de recherche financé par le Conseil de recherches en sciences humaines du Canada (2016-2019) portant sur les stratégies de créacollage numérique. Les résultats présentés ici proviennent d'une analyse de données recueillies à l'aide de certaines questions tirées d'un questionnaire plus large. L'objectif étant de décrire comment les compétences de référencement documentaire des étudiants se développent pendant leur parcours universitaire, nous nous sommes intéressés à trois dimensions, à savoir : 1) l'utilisation de logiciels de référencement; 2) l'efficacité personnelle en référencement; 3) la résolution de problèmes en matière de référencement.

\section{Échantillon}

L'échantillon volontaire total de cette recherche est composé de 1599 étudiants universitaires provenant de six établissements universitaires du Québec. Pour la présente étude, n'ont été retenus pour fins d'analyse que les participants qui ont entièrement répondu à toutes les questions de la section portant sur le référencement documentaire. Ainsi, le sous-échantillon de participants aux fins de cet article comprend 810 étudiants universitaires, dont 640 femmes et 170 hommes. Les participants se subdivisent en trois grands groupes de programmes universitaires, à savoir : $33 \%$ proviennent de programmes du secteur de l'éducation, $28 \%$ proviennent de programmes du secteur des sciences et génie et $39 \%$ proviennent du secteur des sciences sociales et sciences de la gestion. L'âge moyen se situe entre 21 et 24 ans. En ce qui concerne le nombre d'années universitaires complétées, $8,1 \%$ n'ont pas encore complété une année, $34,2 \%$ ont complété une année, $19,1 \%$ ont complété deux années, 15,8 \% ont complété trois années, $12,3 \%$ ont complété quatre ou cinq années et $10,5 \%$ ont complété six années ou plus.

\section{Instrument de collecte des données}

Le questionnaire autoadministré en ligne comprenait 66 questions réparties en 7 sections: connaissances préalables $(n=4)$, connaissances universitaires $(n=2)$, compétences informationnelles $(n=14)$, compétences rédactionnelles $(n=15)$, compétences de référencement documentaire $(n=9)$, connaissances du plagiat $(n=11)$ et questions démographiques $(n=11)$. Ce questionnaire a été développé selon une démarche en sept étapes proposée par Dussault, Valois et Frenette (2007) et a été validé par la suite (Peters, Vincent, Fontaine, Fiset-Vincent, 2018). En effet, plusieurs versions du questionnaire ont été soumises à un comité d'experts ainsi que mises à l'essai auprès d'étudiants universitaires. L'échelle de réponse de type Likert à sept crans est utilisée afin de permettre un choix nuancé de réponses : toujours (valeur $=7$ ), très souvent (valeur $=6$ ), souvent (valeur $=5$ ), à l'occasion (valeur $=4)$, rarement (valeur $=3$ ), très rarement (valeur $=2$ ), jamais (valeur $=1$ ), ainsi que l'option «ne s'applique pas ». Les objectifs du questionnaire sont d'établir les stratégies de créacollage numérique utilisées par les étudiants universitaires ainsi que d'explorer les facteurs qui influencent l'utilisation ou la non-utilisation de ces stratégies. La durée du questionnaire était d'environ 15 minutes.

\section{Procédures de recrutement et de collecte de données}

La collecte de données a été réalisée à l'automne 2017. Le projet a d'abord obtenu un certificat éthique de toutes les universités participantes. Des étudiants de six universités ont reçu un courriel de la part de leur administration modulaire ou facultaire les invitant à remplir le questionnaire en ligne. En cliquant sur le lien menant au questionnaire, chaque participant devait 
lire et signer le formulaire de consentement. À la suite de cela, les participants avaient accès au questionnaire et devaient remplir chaque section de manière anonyme. Lorsque le questionnaire était rempli, les participants obtenaient des instructions afin de fermer sécuritairement leur session. Le questionnaire en ligne a été produit sur LimeSurvey et géré sur un serveur informatique de l'Université du Québec en Outaouais (UQO). Les données numériques ont été recueillies de façon anonyme puis enregistrées et conservées sur les serveurs sécurisés fournis par l'UQO.

\section{Méthode d'analyse des données}

Une analyse factorielle exploratoire a été effectuée avec les questions sur le référencement documentaire afin de les regrouper en domaines de savoirs liés au référencement documentaire. Par la suite, ces domaines ont été mis en relation avec le nombre d'années complétées à l'université. Des analyses statistiques descriptives et corrélatives de même qu'une analyse de variance multivariée ont complété l'analyse des données.

\section{Résultats}

Le tableau 1 présente l'analyse factorielle exploratoire effectuée à l'aide d'une méthode d'extraction de type maximum de vraisemblance avec rotation Varimax et calculée pour les 810 participants ayant répondu aux neuf questions. L'analyse a permis de dégager une solution en trois facteurs pour $61 \%$ de la variance totale expliquée. La mesure de précision de l'échantillonnage de Kaiser-Meyer-Olkin (KMO) indique un indice de 0,63 et le test de sphéricité de Bartlett indique une valeur de khi-deux de 2522,52 ( $\mathrm{ddl}=36 ; p<0,0001)$. Une question a été retranchée en raison du lien trop faible qu'elle entretenait avec les trois facteurs retenus. Le tableau 1 présente la solution factorielle.

Bien que la proportion de la variance totale expliquée de l'analyse factorielle exploratoire soit légèrement supérieure à $60 \%$, cette valeur est généralement acceptable en sciences humaines (Hair, Black, Babin, Anderson et Tatham, 2006). Il en va de même avec l'indice KMO qui atteint le seuil d'acceptabilité de 0,6 avec le test de Bartlett, qui rejette l'hypothèse de sphéricité $(p<0,001)$ assurant que les données ne sont pas toutes indépendantes dans la population (Hair et al., 2006).

Les trois facteurs établis représentent trois domaines de savoirs liés au référencement documentaire, soit: l'utilisation de logiciels, l'efficacité personnelle en lien avec le référencement et la résolution de problèmes de référencement. Le tableau 2 présente les statistiques descriptives de même que les analyses corrélatives entre les trois domaines et le nombre d'années complétées. Un test de Kolmogorov-Smirnov a été effectué pour vérifier et attester la distribution normale de l'ensemble des valeurs. La moyenne et l'écart type $(\sigma)$ de chacun de ces facteurs sont les suivants : 2,32 $(\sigma=2,04)$ pour l'utilisation de logiciels, 4,86 $(\sigma=1,05)$ pour l'efficacité personnelle en référencement et 2,57 $(\sigma=1,40)$ pour la résolution de problèmes de référencement. Le tableau 2 présente également les moyennes et les écarts types de chacun des trois facteurs en lien avec six regroupements d'années universitaires complétées, c'est-à-dire de moins d'une année complétée à plus de six années complétées (valeurs [0], [1], [2], [3], [4-5], [6 et plus]). Enfin, un coefficient de corrélation entre les domaines de savoir et les années d'université complétées indique une corrélation de 0,23 $(p<0,001)$ pour l'utilisation de logiciels, de $0,18(p<0,001)$ pour l'efficacité personnelle en référencement et de $-0,03$ (non significatif) pour la résolution de problèmes de référencement. 
Tableau 1

Analyse factorielle des compétences de référencement documentaire

Facteurs et compétences

12

23

\section{Utilisation de logiciels de gestion de données bibliographiques}

- J'utilise un logiciel de gestion de données bibliographiques (EndNote, Zotero, Mendeley, etc.) pour générer automatiquement mes listes de références dans un $\quad 0,94$ logiciel de traitement de texte pour mes travaux.

- J'utilise un logiciel de gestion de données bibliographiques (EndNote, Zotero, 0,98 Mendeley, etc.) pour gérer mes références.

\section{Efficacité personnelle en lien avec le référencement}

- Je sais comment référencer divers types de sources numériques (page Web, blogue, vidéo, diaporama, etc.).

- Je me sens confiant lorsque je fais une bibliographie pour mes travaux.

- Je sais ce qui est admissible ou pas dans une liste de références pour mes travaux universitaires (documents trouvés, documents cités dans le texte, documents lus, mais non cités).

- J'utilise le guide de rédaction des références de mon programme d'études ou propre à ma discipline.

\section{Résolution de problèmes de référencement}

- Lorsque j'ai un problème avec mes références, je demande de l'aide aux bibliothécaires.

- Je consulte de l'information en ligne (site Web, vidéo, capsule, etc.) pour savoir comment créer une liste de références pour mes travaux.

Afin de mieux faire ressortir la relation entre les items et le facteur auquel ils appartiennent, les valeurs inférieures à 0,25 ont été supprimées du tableau.

\section{Tableau 2}

Moyennes et écarts types (ET) des domaines de savoirs et corrélations entre ces domaines et le nombre d'années universitaires complétées

\begin{tabular}{|c|c|c|c|c|c|c|c|}
\hline \multirow[t]{2}{*}{$\begin{array}{c}\text { Années } \\
\text { complétées }\end{array}$} & \multirow[t]{2}{*}{$n$} & \multicolumn{2}{|c|}{ Utilisation de logiciels } & \multicolumn{2}{|c|}{ Efficacité personnelle } & \multicolumn{2}{|c|}{$\begin{array}{l}\text { Résolution de } \\
\text { problèmes }\end{array}$} \\
\hline & & Moyenne & $\sigma$ & Moyenne & $\sigma$ & Moyenne & $\sigma$ \\
\hline 0 & 66 & 1,60 & 1,32 & 4,50 & 1,27 & 3,02 & 1,44 \\
\hline 1 & 277 & 2,17 & 1,95 & 4,73 & 1,31 & 2,58 & 1,46 \\
\hline 2 & 155 & 1,90 & 1,74 & 4,83 & 1,29 & 2,31 & 1,22 \\
\hline 3 & 128 & 2,36 & 1,96 & 4,99 & 1,34 & 2,62 & 1,38 \\
\hline 4 et 5 & 100 & 2,63 & 2,37 & 5,03 & 1,15 & 2,59 & 1,43 \\
\hline 6 et plus & 84 & 3,77 & 2,57 & 5,25 & 1,21 & 2,60 & 1,45 \\
\hline Total & 810 & 2,32 & 2,04 & 4,86 & 1,05 & 2,57 & 1,40 \\
\hline$r$ & 810 & \multicolumn{2}{|c|}{$0,23 * * *$} & \multicolumn{2}{|c|}{$0,18^{* * *}$} & \multicolumn{2}{|c|}{$-0,03$} \\
\hline
\end{tabular}

Les valeurs varient de 1 (très peu compétent ou jamais) à 7 (très compétent ou toujours). $\sigma:$ écart-type $\quad * * *: p<0,001$

Une analyse de variance multivariée indique une différence significative entre les regroupements d'années universitaires complétées pour chacun des domaines. Plus précisément, une analyse comparative à l'aide de tests post hoc de Bonferroni indique que l'utilisation de logiciels est 
significativement plus fréquente chez les étudiants qui ont cumulé quatre années ou plus d'études universitaires comparativement aux étudiants qui ont complété entre zéro et trois années d'université $([0,1,2,3]<[4-5,6$ et plus $], p<0,001)$. Concernant l'efficacité personnelle, les résultats indiquent une différence significative entre l'entrée à l'université ( 0 année complétée) et les étudiants ayant complété quatre années ou plus ([0]< $[4-5,6$ et plus], $p<0,001)$. Enfin, pour les compétences de résolution de problèmes, bien qu'il existe une différence statistiquement significative entre 0 année complétée et 2 années complétées ( $[0]>[2], p<0,05)$, les résultats ne démontrent aucune autre différence significative entre les regroupements d'années complétées à l'université.

Enfin, une analyse de variance multivariée a été faite entre la moyenne des trois domaines de savoirs et les trois regroupements de programmes universitaires, à savoir : éducation, sciences et génie, sciences sociales et de la gestion. L'analyse de tests post hoc de Bonferroni indique que la fréquence d'utilisation des logiciels de gestion de données bibliographiques du groupe éducation est significativement plus faible $(p<0,01)$ que celle des groupes sciences et génie et sciences sociales et de la gestion. Pour les deux autres domaines de savoirs, aucune différence significative n'a été observée entre les trois regroupements de programmes.

\section{Interprétation et discussion}

\section{Utilisation de logiciels}

Les étudiants débutants utilisent très peu les logiciels de gestion de données bibliographiques comparativement aux étudiants ayant complété plusieurs années à l'université. Ces résultats vont dans le même sens que ceux de Salem et Fehrmann (2013) qui suggèrent que les bénéfices de l'utilisation de logiciels de gestion de données bibliographiques ne sont pas compensés par les efforts d'appropriation de ceux-ci. Speare (2018) indique que même si les étudiants qui entrent à l'université ont de bonnes aptitudes technologiques, ils ne sont pas forcément prêts à investir le temps nécessaire pour utiliser les logiciels, car ils y arrivent avec des outils plus simples qu'ils connaissent déjà, dont les logiciels de traitement de texte.

Par ailleurs, les résultats ont généralement de faibles valeurs si l'on considère que la moyenne plus élevée se situe entre «rarement » et «à l'occasion » pour les étudiants ayant complété six années ou plus d'études universitaires. Il est possible que l'utilisation de logiciels de gestion de données bibliographiques ne soit pas encouragée selon les programmes ou les disciplines enseignés ou que les avantages de leur utilisation ne soient pas bien démontrés. De plus, Park et al. (2011) indiquent que les professeurs ont souvent des exigences qui ne sont pas uniformes en matière de référencement. Certains iront même jusqu'à considérer que le fait de ne pas référencer correctement n'est pas une erreur très grave, pourvu que toutes les sources soient présentes dans la bibliographie (Park et al., 2011). D'autres vont laisser le libre choix à leurs étudiants d'utiliser des normes de présentation ou un style particulier. Cela fait en sorte que les professeurs n'ont pas toujours toutes les connaissances des différents styles ou normes pour corriger ou indiquer les erreurs de référencement commises par leurs étudiants (Kargbo, 2010).

Enfin, on peut émettre l'hypothèse que la recherche et l'intégration de données scientifiques dans les travaux à l'intérieur des activités de $1^{\mathrm{er}}$ cycle ne sont pas suffisamment importantes pour favoriser l'utilisation de logiciels de gestion de données bibliographiques. Par ailleurs, l'activité de recherche et d'intégration de données scientifiques dans les travaux augmente de manière significative aux cycles supérieurs, créant un contexte plus favorable à l'utilisation de logiciels de gestion de données bibliographiques. Il devient ainsi compréhensible que les étudiants ayant 
complété moins de quatre ans d'université aient eu moins de possibilités de réaliser de grands travaux exigeant une gestion importante de données bibliographiques. Dans ce contexte, les expériences de cycles supérieurs sont susceptibles de motiver les étudiants à s'approprier de nouveaux outils plus performants afin de gérer plus efficacement l'ampleur des travaux à réaliser.

\section{Efficacité personnelle}

De façon générale, les résultats indiquent que les étudiants qui entament un parcours universitaire se sentent moins efficaces en ce qui concerne leurs aptitudes de référencement comparativement aux étudiants plus avancés. Par contre, la perception des étudiants est assez élevée dès l'entrée à l'université, si l'on en juge par une moyenne qui se situe à 4,50/7 et progresse positivement tout au long de l'expérience universitaire. Ces résultats peuvent s'expliquer par une préparation relativement bonne avant l'entrée à l'université, au collégial ou même au secondaire. Avec l'avènement du renouveau pédagogique, les compétences liées aux méthodes de travail ont été introduites et enseignées dès le primaire (Ministère de l'Éducation, 2006). Les étudiants peuvent avoir la chance de s'approprier des concepts importants en lien avec le référencement et développer ainsi une perception de leur propre efficacité en la matière (Newton, 2016). Le sentiment d'efficacité personnelle en lien avec le référencement étant ainsi positivement élevé dès l'entrée à l'université, il est normal qu'un effet de plafonnement apparaisse et que l'augmentation soit moins significative. D'ailleurs, il faut comparer le groupe des étudiants n'ayant aucune année complétée avec celui ayant complété 4, 5 ou 6 années et plus avant d'obtenir une différence suffisamment grande pour qu'elle soit jugée significative.

Lorsque les étudiants ont complété quatre années d'université et plus, leur expérience de recherche aux cycles supérieurs est susceptible d'influencer la perception de leur propre efficacité en ce qui concerne le référencement documentaire. De plus, les expériences d'utilisation de logiciels de gestion de données bibliographiques peuvent s'ajouter et expliquer un sentiment de confiance plus élevé envers leurs compétences de référencement.

\section{Résolution de problèmes}

Les résultats ne suggèrent pas de lien entre les années d'université complétées et la recherche de solutions à des problèmes de référencement. Ils indiquent que peu importe le nombre d'années complétées à l'université, lorsqu'un étudiant rencontre un problème, il dispose de plusieurs stratégies qu'il va exploiter afin de le résoudre. Les résultats ont tout de même fait ressortir que les étudiants débutants utilisent davantage les ressources en ligne et les bibliothécaires que leurs pairs ayant terminé deux années d'université. Ce constat peut s'expliquer par le fait que les premières années d'université sont l'occasion de s'approprier les normes ou les styles de référencement. Une fois les problèmes résolus, les contextes d'apprentissage peuvent être relativement les mêmes au cours des deux ou trois premières années d'université. Lorsque l'étudiant chemine aux cycles supérieurs, il fait face à de nouveaux problèmes qu'il résout avec les stratégies utilisées au cours des premières années universitaires. Lorsque ces stratégies ont démontré leur efficacité, elles peuvent être réutilisées et influer positivement sur le sentiment d'efficacité personnelle.

\section{Conclusion}

Les compétences de référencement documentaire sont essentielles dans le contexte des études universitaires, notamment dans le cadre de la promotion de l'intégrité intellectuelle et de 
l'évitement du plagiat. Parmi les savoirs qui facilitent le référencement documentaire, l'utilisation de logiciels de gestion de données bibliographiques est un outil efficace en lien avec la rédaction des travaux universitaires. Toutefois, la présente étude suggère que l'utilisation s'opère après plusieurs années complétées à l'université. Sans que cette étude puisse le vérifier avec exactitude, il est possible d'émettre l'hypothèse que l'utilisation des logiciels se fait au moment où les étudiants accèdent aux cycles supérieurs. En effet, de façon générale, les travaux de $1^{\text {er }}$ cycle n'exigent pas de gérer une grande quantité de sources, ce qui fait que l'utilisation des logiciels de gestion de données bibliographiques exige plus de temps et d'énergie qu'elle peut en faire gagner.

Ce constat est aussi cohérent avec le sentiment d'efficacité personnelle des étudiants au regard du référencement. En effet, les étudiants qui entrent à l'université ont une grande confiance en leurs aptitudes de référencement. Cette confiance s'établit en lien avec les outils qu'ils maîtrisent ou utilisent de manière régulière. Lorsque la nécessité d'un référencement plus efficace se fait sentir, cela crée un contexte favorable à l'appropriation des logiciels et permet du même coup d'améliorer le sentiment d'efficacité personnel au regard des compétences de référencement documentaire. Quant aux compétences de résolution de problèmes liées au référencement, les stratégies semblent être présentes dans l'ensemble de la formation universitaire, que l'étudiant soit débutant ou avancé dans son cheminement universitaire.

Cette étude comporte plusieurs limites qu'il importe de préciser. D'abord, elle a été effectuée dans un cadre plus large sur la description et l'enseignement de stratégies de créacollage numérique à l'université. Comme les compétences de référencement documentaire ne concernent pas uniquement le numérique, cette étude ne comportait pas de grand plan. Également, plusieurs participants de l'échantillon ont été rejetés en raison de non-réponses. Bien que la durée du questionnaire n'ait été que de 15 minutes, il faut se poser la question de savoir si le format ou les types de questions n'ont pas attiré un type particulier de répondants. Comme le questionnaire était rempli en ligne, il n'était pas possible de donner des informations additionnelles ou de veiller à saisir les bonnes informations aux bons moments afin d'éviter des erreurs, comme entrer la date du jour au lieu de la date de naissance. Ensuite, les différents regroupements pour les années complétées à l'université ne tenaient pas compte du cycle, des disciplines et des programmes complétés. Ainsi, le groupe d'étudiants qui indiquait par exemple six années ou plus pouvait inclure des participants qui étaient en train d'effectuer un deuxième baccalauréat, et ce, peut-être après avoir complété une maîtrise.

D'autres recherches sont nécessaires, notamment pour mieux comprendre les relations entre les compétences de référencement documentaire et les exigences scientifiques des programmes d'études. En effet, les programmes d'études universitaires n'ont pas tous des exigences et des besoins équivalents en matière de compétence de référencement. De plus, à la lumière des résultats de cette étude, les différents cycles universitaires devront être mieux contrôlés afin d'étayer avec plus de précision le développement des compétences de référencement. En effet, les travaux exigés aux $2^{\mathrm{e}}$ et $3^{\mathrm{e}}$ cycles à l'université ne sont pas de même nature que ceux du $1^{\mathrm{er}}$ cycle. Par exemple, rédiger un mémoire ou une thèse requiert des compétences nettement plus développées sur le plan du référencement documentaire.

Enfin, il est possible de formuler une recommandation à la suite de la présente étude. Il serait pertinent que tous les étudiants qui entrent à l'université soient, rapidement et systématiquement, formés à l'utilisation de logiciels de gestion de base de données bibliographiques et aux compétences de référencement documentaire (Beauchemin-Roy, Cadieux et Peters, 2019). Cette 
formation leur permettrait d'être encore plus confiants et performants, tout au long de leurs études universitaires, lors de la rédaction de leurs travaux.

\section{Références}

Artino, A. R. Jr. (2012). Academic self-efficacy: From educational theory to instructional practice. Perspectives on Medical Education, 1(2), 76-85.

https://doi.org/10.1007/s40037-012-0012-5

Bandura, A. (1977). Self-efficacy: Toward a unifying theory of behavioral change. Psychological Review, 84(2), 191-215. doi:10.1037/0033-295X.84.2.191 Récupéré du site du National Consortium for Multicultural Education, Université Stanford: http://culturalmeded.stanford.edu

Beauchemin-Roy, S., Cadieux, A. et Peters, M. (2019, mars). Use of referencing software among university students: Experience is key. Communication présentée au $13^{\mathrm{e}}$ Congrès annuel International Technology, Education and Development Conference, Valence, Espagne. Récupéré du site du Groupe de recherche sur l'intégrité académique, Université du Québec en Outaouais : http://w4.uqo.ca/mpeters

Brown, C. A., Dickson, R., Humphreys, A.-L., McQuillan, V. et Smears, E. (2008). Promoting academic writing/referencing skills: Outcome of an undergraduate e-learning pilot project. British Journal of Educational Technology, 39(1), 140-156. doi:10.1111/j.14678535.2007.00735.x

Cadieux, A., Morinière, G. et Simonnot, B. (2018). Compétences de référencement documentaire: quoi, pourquoi et comment référencer? [diaporama]. Webinaire 2017-2018, Groupe de recherche sur l'intégrité académique, Université du Québec en Outaouais. Récupéré du site du Groupe : http://w4.uqo.ca/mpeters

Couture, M. (2010). Les références aux documents en ligne dans les textes scientifiques. Revue internationale des technologies en pédagogie universitaire, 7(2), 6-19.

https://doi.org/10.18162/ritpu.2010.178

Couture, M. (2017, 17 mai). Normes bibliographiques. Adaptation française des normes de l'APA. Récupéré le 11 novembre 2018 du site de l'auteur : http://teluq.ca/ mcouture

Cuschieri, S., Grech, V. et Calleja, N. (2019). WASP (Write a scientific paper): The use of bibliographic management software. Early Human Development, 128, 118-119. doi:10.1016/j.earlhumdev.2018.09.012

Dahlstrom, E. et Bichsel, J. (2014). ECAR study of undergraduate students and information technology, 2014. Récupéré de http://educause.edu

Dahlstrom, E., de Boor, T., Grunwald, P. et Vockley, M. (2011). ECAR national study of undergraduate students and information technology, 2011. Récupéré de http://educause.edu

Dussault, M., Valois, P. et Frenette, E. (2007). Validation de l'échelle de leadership transformatif du directeur d'école. Psychologie du travail et des organisations, 13(2), 37-52.

Francese, E. (2013). Usage of reference management software at the University of Torino. Italian Journal of Library, Archives and Information Science, 4(2), 145-170. https://doi.org/10.4403/jlis.it-8679 
Guerrin, B. (2012). Albert Bandura et son œuvre. Recherche en soins infirmiers, 2012/1(108), 106-116. https://doi.org/10.3917/rsi.108.0106

Hair, J. F., Black, W. C., Babin, B. J., Anderson, R. E. et Tatham, R. L. (2006). Multivariate data analysis ( $6{ }^{\mathrm{e}}$ éd.). New Jersey, É.-U. : Prentice Hall.

Kargbo, J. A. (2010). Undergraduate students' problems with citing references. The Reference Librarian, 51(3), 222-236. doi:10.1080/02763871003769673

Ministère de l'Éducation. (2006). Programme de formation de l'école québécoise. Éducation préscolaire, enseignement primaire. Québec : Gouvernement du Québec. Récupéré du site du ministère de l'Éducation et de l'Enseignement supérieur :

http://education.gouv.qc.ca

Monney, N., Peters, P., Boies, T. et Raymond, D. (2019). Évaluer la compétence de référencement documentaire chez des étudiants de premier cycle universitaire : pratiques déclarées d'enseignants universitaires. Revue internationale des technologies en pédagogie universitaire, 16(2), 39-55. https://doi.org/10.18162/ritpu-2019-v16n2-05

Newton, P. (2016). Academic integrity: A quantitative study of confidence and understanding in students at the start of their higher education. Assessment and Evaluation in Higher Education, 41(3), 482-497. https://doi.org/10.1080/02602938.2015.1024199

Park, S., Mardis, L. A. et Ury, C. J. (2011). I've lost my identity - oh, there it is... in a style manual: Teaching citation styles and academic honesty. Reference Services Review, 39(1), 42-57. doi:10.1108/00907321111108105

Peters, M. (2015). Enseigner les stratégies de créacollage numérique pour éviter le plagiat au secondaire. Revue canadienne d'éducation, 38(3). Récupéré de http://journals.sfu.ca/cje

Peters, M., Vincent, F., Fontaine, S. et Fiset-Vincent, C. (2018). Validation d'un questionnaire sur les stratégies de créacollage numérique d'étudiants universitaires québécois. Revue internationale des technologies en pédagogie universitaire, 15(1), 45-60. https://doi.org/10.18162/ritpu-2018-v15n1-05

Peters, M., Vincent, F., Gervais, S., Morin, S. et Pouliot, J.-P. (2019). Les stratégies de créacollage numérique et les compétences requises pour les mobiliser. Dans T. Karsenti (dir.), Le numérique en éducation. Pour développer des compétences (p. 159-180). Québec, Canada : Presses de l'Université du Québec.

Ram, S. et Anbu K., J. P. (2014). The use of bibliographic management software by Indian library and information science professionals. Reference Services Review, 42(3), 499-513. doi:10.1108/RSR-08-2013-0041

Robert, T. S. (2008). Student plagiarism in an online world: Problems and solutions. Hershey, PA : Information Sciences Reference. doi:10.4018/978-1-59904-801-7

Salem, J. et Fehrmann, P. (2013). Bibliographic management software: A focus group study of the preferences and practices of undergraduate students. Public Services Quarterly, 9(2), 110-120. doi:10.1080/15228959.2013.785878

Speare, M. (2018). Graduate student use and non-use of reference and PDF management software: An exploratory study. The Journal of Academic Librarianship, 44(6), 762-774. doi:10.1016/j.acalib.2018.09.019 
Staats, A. W. (1996). Behavior and personality. New York, É.-U. : Springer.

Tsai, C.-Y. (2018). Improving students' understanding of basic programming concepts through visual programming language: The role of self-efficacy. Computers in Human Behavior, 95, 224-232. doi:10.1016/j.chb.2018.11.038 Manuscrit récupéré du profil de l'auteur dans ResearchGate : http://researchgate.net/profile/Chun_Yen_Tsai

Vardi, I. (2012). Developing students' referencing skills: A matter of plagiarism, punishment and morality or of learning to write critically? Higher Education Research \& Development, 31(6), 921-930. doi:10.1080/07294360.2012.673120

Vincent, F. et Cadieux, A. (2019). Comment enseigner la paraphrase et la citation au postsecondaire? Apprendre à rédiger pour assurer l'intégrité académique [diaporama]. Webinaire 2018-2019, Groupe de recherche sur l'intégrité académique, Université du Québec en Outaouais. Récupéré du site du Groupe : http://w4.uqo.ca/mpeters

Wang, Y. et Chiew, V. (2010). On the cognitive process of human problem solving. Cognitive Systems Research, 11(1), 81-92. doi:10.1016/j.cogsys.2008.08.003 Récupéré du site de l'International Institute of Cognitive Informatics and Cognitive Computing (ICIC), Université de Calgary : http://ucalgary.ca/icic 